\title{
Searches for Hyperbolic Extra Dimensions at the LHC
}

\author{
Henrik MELBÉUS*† \\ Department of Theoretical Physics, Royal Institute of Technology (KTH), 10691 Stockholm, \\ Sweden \\ E-mail: melbeus@kth.se
}

In this poster, we present a model of large extra dimensions where the internal space has the geometry of a hyperbolic disc. Compared with the ADD model, this model provides a more satisfactory solution to the hierarchy problem between the electroweak scale and the Planck scale, and it also avoids constraints from astrophysics. Since there is no known analytic form of the Kaluza-Klein spectrum for our choice of geometry, we obtain a spectrum based on a combination of approximations and numerical computations. We study the possible signatures of our model for hadron colliders, especially the LHC, where the most important processes are the production of a graviton together with a hadronic jet or a photon. We find that for the case of hadronic jet production, it is possible to obtain relatively strong signals, while for the case of photon production, this is much more difficult.

Identification of dark matter 2008

August 18-22, 2008

Stockholm, Sweden

\footnotetext{
*Speaker.

${ }^{\dagger}$ In collaboration with Tommy Ohlsson.
} 


\section{INTRODUCTION AND THE HIERARCHY PROBLEM}

One of the main motivations for large extra dimensions is that they provide a solution to the so-called hierarchy problem between the electroweak scale $M_{\mathrm{ew}} \simeq 100 \mathrm{GeV}$ and the Planck scale $M_{\mathrm{Pl}} \equiv G_{N}^{-1 / 2} \simeq 10^{19} \mathrm{GeV}$. Theoretically, $M_{\mathrm{ew}}$ is expected to obtain loop corrections of order $M_{\mathrm{Pl}} \gg M_{\mathrm{ew}}$. Therefore, a miraculous cancellation is needed to keep $M_{\mathrm{ew}}$ at the order of $100 \mathrm{GeV}$. The so-called ADD (Arkani-Hamed, Dimopoulos, and Dvali) model [1] provides a very elegant solution to this problem. However, this solution is not completely satisfactory, since it turns out to be just a reformulation of the problem, which means that the ADD model still suffers from the hierarchy problem. In models with hyperbolic geometry [2], this problem might be remedied.

\section{THE HYPERBOLIC DISC MODEL}

The model that we consider is similar to the ADD model, with the only exception that the internal space is a two-dimensional hyperbolic disc, with constant negative curvature $v$ [3]. The SM fields are assumed to be confined to a four-dimensional brane, while gravity alone probes the extra dimensions. See Fig. 1 for an illustration.

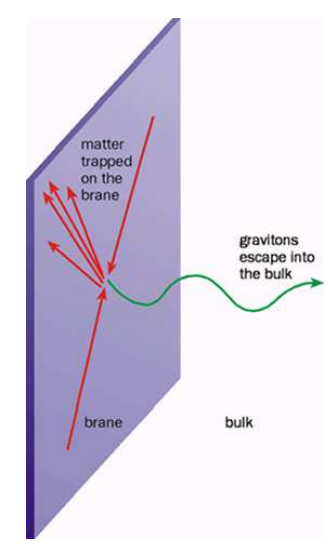

Figure 1: An illustration of the brane and the extra dimensions. The figure has been adopted from [ 4 ].

In the ADD model, the Planck scale $M_{\mathrm{Pl}}$ is replaced by a new mass scale $M_{*}$. The two scales are related through the equation $M_{\mathrm{Pl}}^{2}=V M_{*}^{4}$. If $V$ is large enough, then $M_{*}$ could be as low as $M_{\mathrm{ew}} \simeq 1 \mathrm{TeV}$, hence eliminating the hierarchy problem between $M_{\mathrm{Pl}}$ and $M_{\mathrm{ew}}$. However, in the ADD model, the radius $L \simeq 10^{31 / d} M_{*}$, and thus, a new hierarchy problem is created, which means that the hierarchy problem is only reformulated as the question of why the radius of the internal space is so large compared to the electroweak scale.

The area of a hyperbolic disc with radius $L$ is

$$
V=\frac{4 \pi}{v^{2}} \sinh ^{2}\left(\frac{v L}{2}\right) \approx \frac{\pi}{v^{2}} \exp (v L), \quad v L \gg 1 .
$$

This has the result that, if the internal space is a hyperbolic disc, then it is possible to have $M_{\mathrm{Pl}} \simeq$ $M_{\mathrm{ew}} \simeq L^{-1}$, i.e., no new large hierarchy is introduced, if also $v \simeq M_{*}$. 


\section{COMPARISON WITH THE ADD MODEL}

In the ADD model, there is a single free parameter, which can be taken to be the mass scale $M_{*}$. In the hyperbolic disc model, the curvature $v$ enters as an additional parameter. In the ADD model, the physical results are independent of the position of the brane in the internal space. In the hyperbolic disc model, this is no longer true, and the results depend on the radial position of the brane on the disc. Hence, this position, $\tau_{b}$, is a another free parameter. In total then, there are three free parameters in the hyperbolic disc model.

Since the KK spectrum starts out at $m \approx 0$ in the ADD model, it is important to make sure that there is no overproduction of light KK modes in hot systems, such as supernovae or the early Universe. These considerations place restrictions on the size of $M_{*}$ in the ADD model, in particular for $d=2$, in which case the lower bound is $M_{*} \geq 50 \mathrm{TeV}$. In the hyperbolic disc model, on the other hand, it seems likely that the spectrum starts out at the non-zero value $m=v / 2$. Hence, if $v$ is large enough, then these astrophysical constraints are avoided. A value for $v$ of the same order of magnitude as $M_{*}$ is desirable in order not to introduce a new large hierarchy, and in this case $v$ is by far large enough to avoid the constraints on $M_{*}$.

Finally, while it is possible to find an exact solution for the KK spectrum in the ADD model, this is not possible in the hyperbolic disc model, due to the more complicated geometry. Hence, only approximate results can be obtained for this model.

Thus, we observe that in the hyperbolic disc model, the hierarchy problem can be solved and the astrophysical constraints on $M_{*}$ can be avoided. However, there are additional complications compared to the ADD model, since the parameter space is larger, and since there is no analytic solution for the KK spectrum.

\section{LHC PHENOMENOLOGY}

The KK modes of the graviton couple to all SM fields. We have considered possible production processes of KK gravitons at the LHC. Since each individual KK mode couples very weakly to SM fields, we need to study the production of any kinematically available KK mode. Because of the weak couplings, the graviton is not observed in detectors, and hence, we consider production of a graviton together with some observable particle. At the LHC, there are two interesting reactions: $p+p \rightarrow$ jet $+G$ and $p+p \rightarrow \gamma+G$, which both give rise to missing energy signals. See Fig. 目 for the Feynman diagrams of the two reactions.
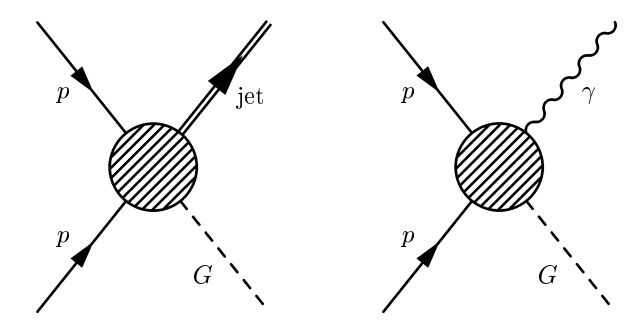

Figure 2: The Feynman diagrams for the reactions $p+p \rightarrow$ jet $+G$ (left) and $p+p \rightarrow \gamma+G$ (right). 


\section{RESULTS}

For both of the reactions mentioned above, we have computed the differential cross sections with respect to the transverse momentum $p_{\mathrm{T}}$ of the outgoing jet/photon, and with respect to $\cos (\theta)$, where $\theta$ is the angle between the beam and the jet/photon. As a reference, we also give the corresponding results for the ADD model, as well as the SM background, which mainly comes from the processes $p+p \rightarrow \mathrm{jet} / \gamma+Z(\rightarrow v \bar{v})$.

In Figs. 3 and 4 , the jet production results that could be observed at the LHC are shown as functions of $p_{\mathrm{T}}$ and $\cos (\theta)$, respectively, whereas in Figs. 5 and 6 , the corresponding results for photon production are shown.
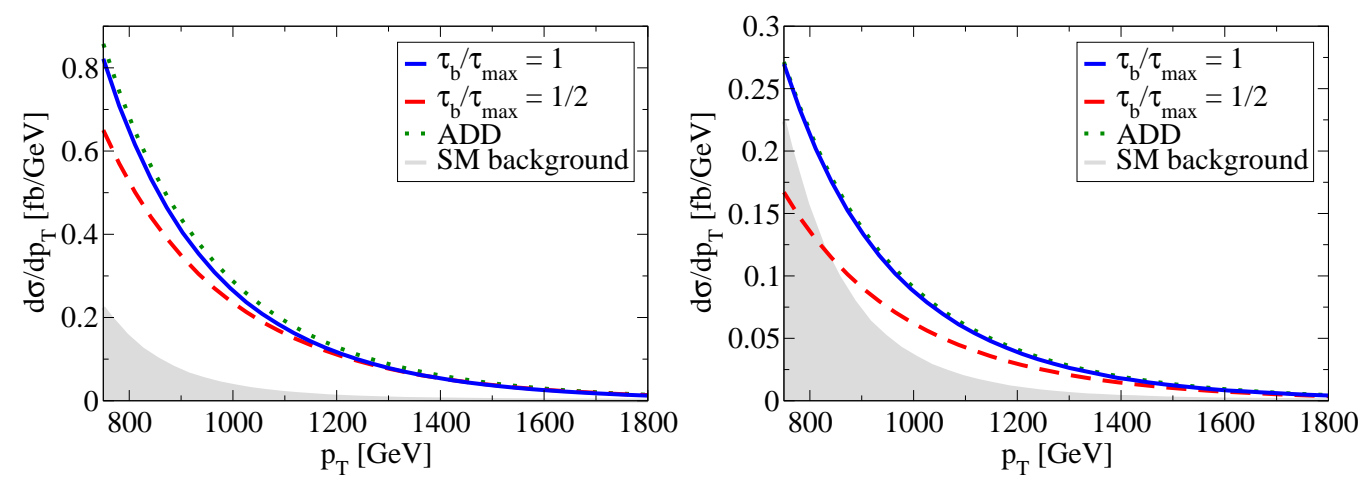

Figure 3: The differential cross section for graviton plus jet production with respect to $p_{\mathrm{T}}$. Left panel: $M_{*}=1.5 \mathrm{TeV}$. Right panel: $M_{*}=2 \mathrm{TeV}$.
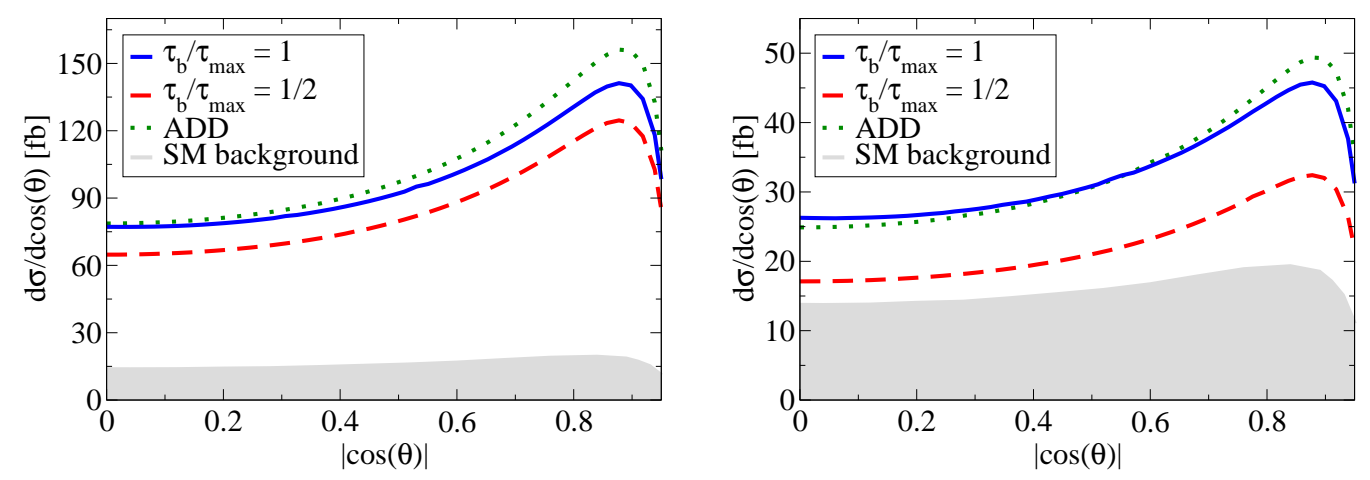

Figure 4: The differential cross section for graviton plus jet production with respect to $\cos (\theta)$. Left panel: $M_{*}=1.5 \mathrm{TeV}$. Right panel: $M_{*}=2 \mathrm{TeV}$.

\section{CONCLUSIONS}

In conclusion, we have found that i) the signals are similar to those of the ADD model, ii) the most promising signal comes from the jet channel, and finally, iii) it is more difficult to obtain an observable signal from the photon channel. 

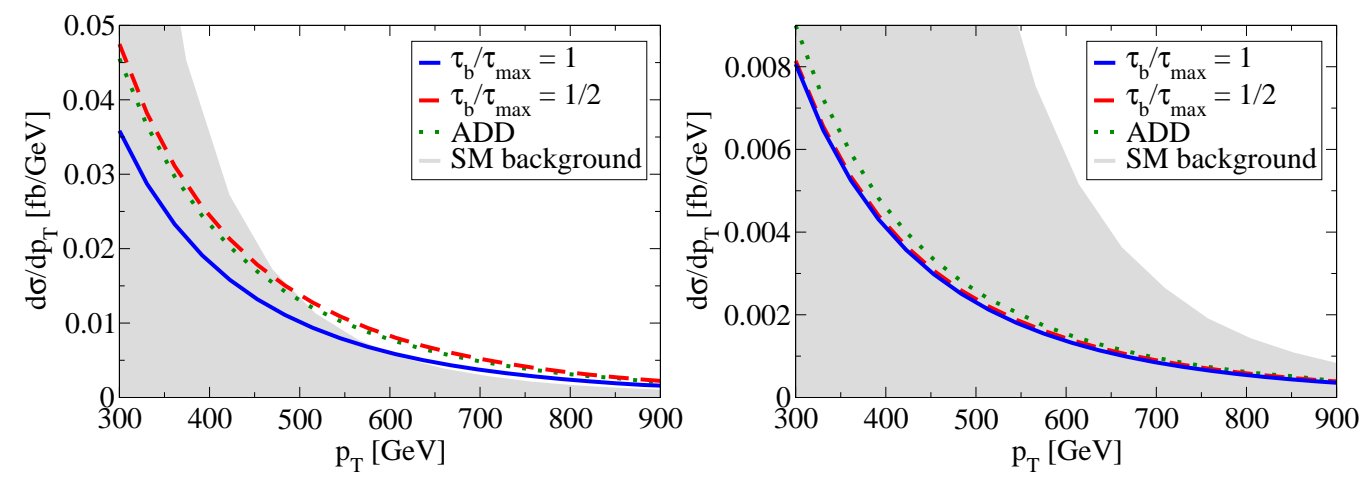

Figure 5: The differential cross section for graviton plus photon production with respect to $p_{\mathrm{T}}$. Left panel: $M_{*}=1 \mathrm{TeV}$. Right panel: $M_{*}=1.5 \mathrm{TeV}$.
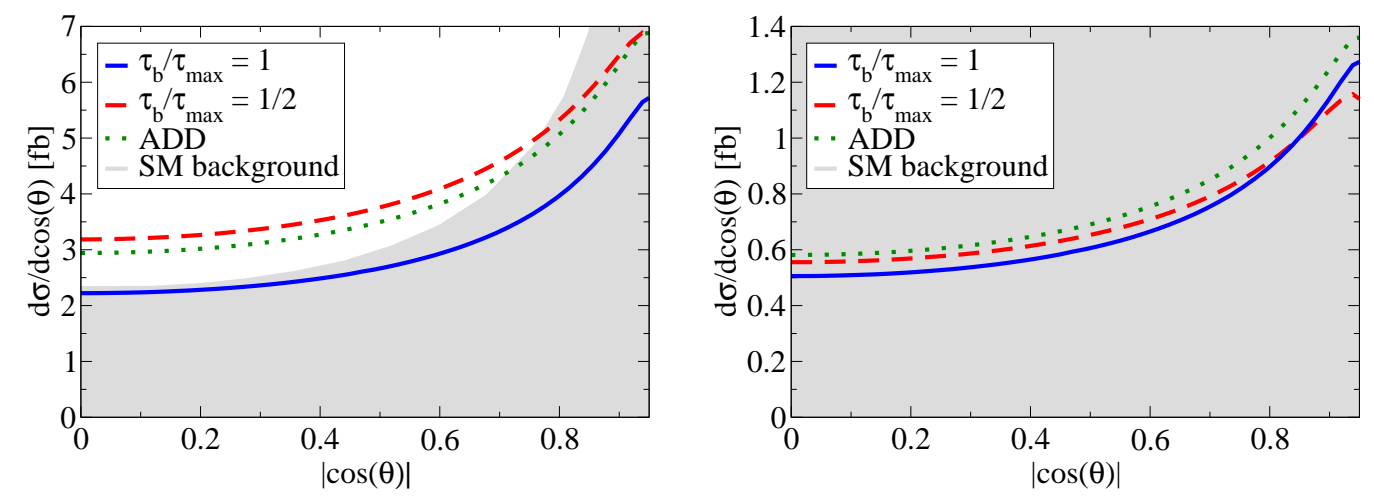

Figure 6: The differential cross section for graviton plus photon production with respect to $\cos (\theta)$. Left panel: $M_{*}=1 \mathrm{TeV}$. Right panel: $M_{*}=1.5 \mathrm{TeV}$.

\section{Acknowledgments}

I would like to thank Tommy Ohlsson for useful collaboration that led to the publication [3] upon which this poster is based. In addition, I would like to thank the organizers of idm 2008 for the possibility to show the poster.

\section{References}

[1] N. Arkani-Hamed, S. Dimopoulos, and G.R. Dvali, The Hierarchy Problem and New Dimensions at a Millimeter, Phys. Lett. B429, 263 (1998), [hep-ph/9803315].

[2] N. Kaloper, J. March-Russell, G.D. Starkman, and M. Trodden, Compact Hyperbolic Extra Dimensions: Branes, Kaluza-Klein Modes, and Cosmology, Phys. Rev. Lett. 85, 928 (2000), [hep-ph/0002001].

[3] H. Melbéus and T. Ohlsson, Searches for hyperbolic extra dimensions at the LHC, J. High Energy Phys. 08, 077 (2008), [arXiv: 0806.1841 [hep-ph] ].

[4] S. Abel and J. March-Russell, The search for extra dimensions, Physics world (November 2000), 38-44 